\title{
31. PLASTICITY CHARACTERISTICS OF THE QUATERNARY SEDIMENTS OF THE GUATEMALAN CONTINENTAL SLOPE, MIDDLE AMERICA TRENCH, AND COCOS PLATE-LEG 67, DEEP SEA DRILLING PROJECT ${ }^{1}$
}

\author{
Richard W. Faas, Department of Geology, Lafayette College, Easton, Pennsylvania
}

\begin{abstract}
The plasticity characteristics of the Quaternary sediments of the Guatemalan continental margin were determined from five sites drilled during Leg 67 of the Deep Sea Drilling Project. The 64 samples analyzed are from various marine environments, including the Cocos Plate, Middle America Trench, and the trench lower slope to midslope of the Guatemalan continental slope. The sediments are primarily hemipelagic muds and trench-fill turbidites and include quantities of siliceous and calcareous biogenic components.

The sediments are generally classified as organic clays of medium to high plasticity, containing micaceous sands and silts, with $14 \%$ classed as inorganic clays of medium to high plasticity.

High sedimentation rates in Quaternary sediments are the result, in part, of sediment gravity flows that depend upon rheological properties, i.e., sediment plasticity. Mudflows and cohesive debris flows appear to be significant downslope transport mechanisms in these highly plastic sediments.
\end{abstract}

\section{INTRODUCTION}

As more interest is focused on active continental margins and their deposits, it is inferred that many of these are areas of downslope sediment transport. Such transport may be rapid and episodic, it may be slow and pervasive; or it may be some combination of the two. Lowe (1979) has indicated the importance of rheology in distinguishing the mass transport mechanisms as fluidal flows or debris flows by their fluid and plastic behavior, respectively. Insofar as this concept has validity, the expected rheological behavior of the sediments accumulating on any particular continental margin should be assessed before any specific process is studied in depth. A measurement of the rheological characteristics of continental margin sediments is readily available in the form of the Atterberg limits.

The purpose of this paper is to present the plasticity characteristics of the Quaternary sediments from the Guatemalan continental margin at each of the Leg 67 sites (excluding Sites 498 and 500) (Fig. 1) and to suggest that these characteristics may be useful in predicting the form of downslope mass transport that may be or may have been operating. The data consist of shipboard measurements of initial water content and Atterberg limits. Laboratory analysis of particle size distribution of the sediment from the same samples enabled a determination of the "activity" of the sediments.

\section{Atterberg Limits}

Atterberg limits (Casagrande, 1948) are based on the concept that a soil is a two-phase system composed of sediment particles and water. The rheological behavior

\footnotetext{
${ }^{1}$ Aubouin, J., von Huene, R., et al., Init. Repts. DSDP, 67: Washington (U.S. Govt. Printing Office).
}

state of such a mixture changes from fluid to plastic to solid as the mixture loses water. The point at which a sediment-water mixture ceases to behave as a liquid is its liquid limit $\left(w_{L}\right)$. Similarly, plastic behavior of such a system ceases at its plastic limit $\left(w_{P}\right)$ and it passes through a semisolid state, determined by its shrinkage limit $\left(w_{S}\right)$. Each limit is the empirical measure of the water content of the soil at that point where its behavior changes from one state to another. The plasticity index $\left(I_{P}\right)$ is a measure of the range of water content through which the sediment exhibits plastic behavior:

$$
I_{P}=w_{L}-w_{P}
$$

The liquidity index $\left(I_{L}\right)$ allows the natural water content of a soil-water system to be compared directly with its Atterberg limits and can be used to predict the consolidation state of a sedimentary section (Skempton, 1970). It is defined as:

$$
I_{L}=\frac{w-w_{P}}{I_{P}}
$$

where: $w=$ initial water content, $w_{P}=$ plastic limit, and $I_{P}=$ plasticity index.

\section{Plasticity Characteristics}

Through the determination of its Atterberg limits, a sediment can be described and classified into various degrees of high, medium, and low plasticity. This classification, developed by Casagrande (1948), utilizes the relationship between the liquid limit $\left(w_{L}\right)$ and the plasticity index $\left(I_{P}\right)$ of a sample to determine its position within the total range of plastic behavior. The A-line in Figure 2 is an empirical boundary separating inorganic clays (above the line) and organic clays and inorganic silts (below the line). 


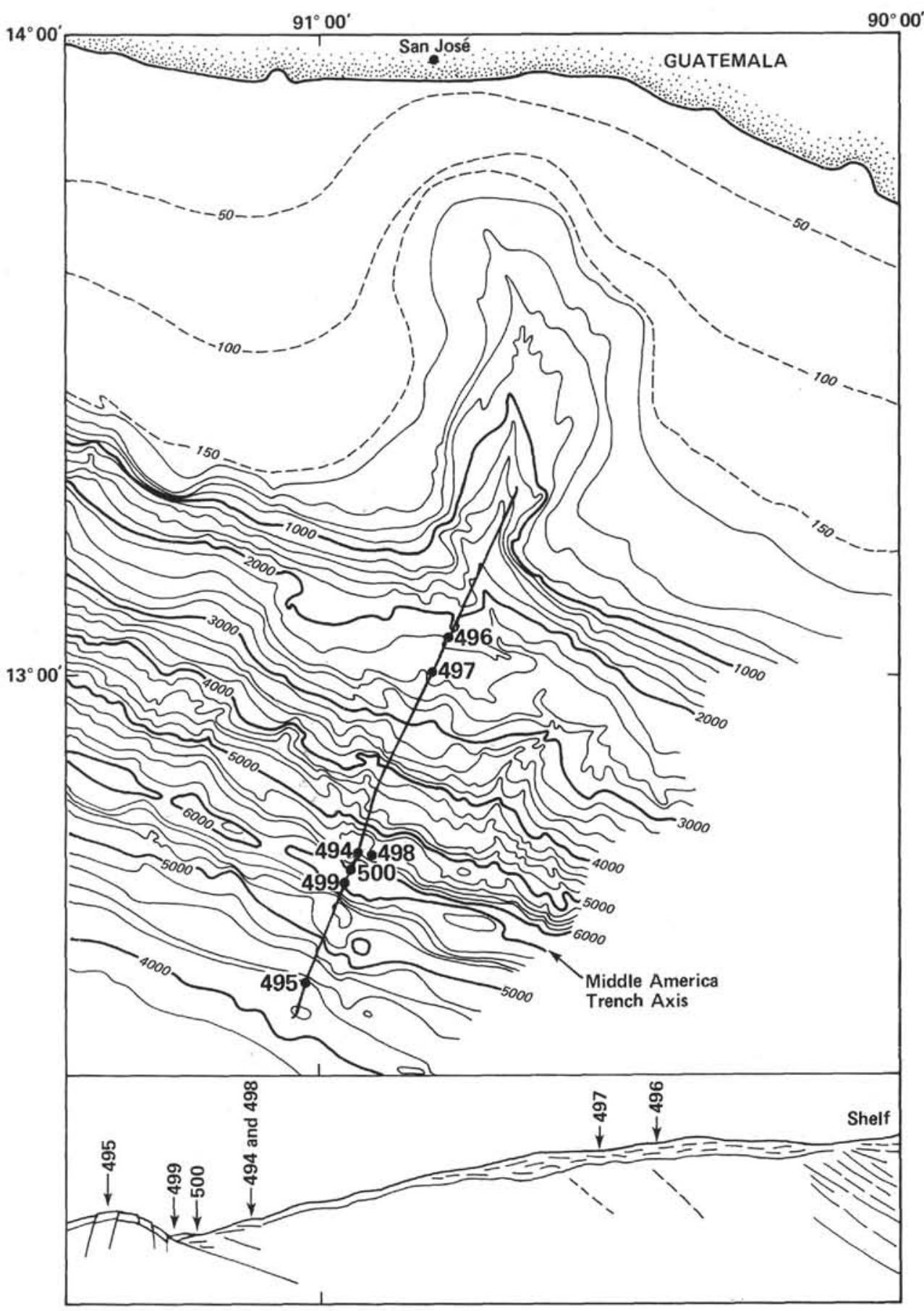

Figure 1. Location map-Leg 67 Sites.

Keller, Lambert, and Bennett (1979) utilized this technique to map the continental slope deposits between the Hydrographer Canyon and Cape Hatteras into four groups of sediment with increasing plasticity. Davie, Fenske, and Serocki (1978) show the variation in plasticity for different sediment types, including calcareous and siliceous oozes, clay, and volcanic ash from 89 continental margin locations. And Richards and Fager (1980) present data on the plasticity of pelagic clays and radiolarian clay and ooze from the Hatteras Abyssal Plain.

\section{Activity}

Skempton (1953) found that the relationship between the plasticity index and the clay fraction (\% less than 2 $\mu \mathrm{m}$ ) of any particular combination of clay minerals remained essentially constant. Each mixture of clay minerals can therefore be characterized by a single parameter. This he called "activity," defined as:

$$
\text { Activity }\left(A_{c}\right)=\frac{\text { Plasticity Index }}{\text { Clay fraction ( } \% \text { less than } 2 \mu \mathrm{m})}
$$




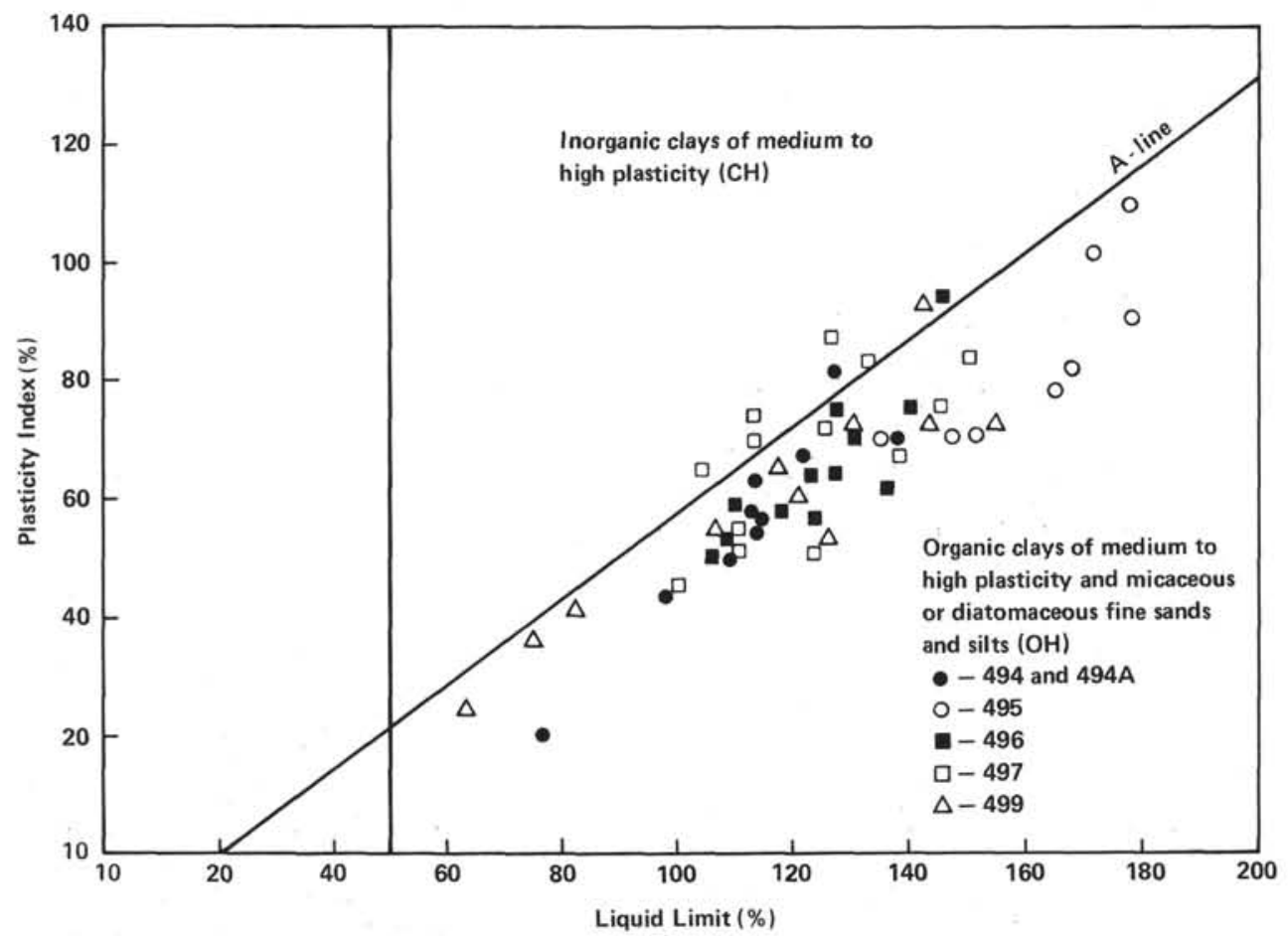

Figure 2. Casagrande Plasticity Chart showing plasticity characteristics of all samples analyzed. (The A-line is an empirical boundary separating inorganic clays from organic clays and inorganic silts. The plasticity index and liquid limit values are based on $\%$ dry wt. of sediment; consequently it is possible to have values greater than $100 \%$-indeed, it is expected.)

Activity represents the surface activity of the clay fraction, such as the increased ion exchange capacity and absorption of water with decreased grain size. Activity of pure clays increases from a low of 0.23 (muscovite) to a high of 6.0 (Na-montmorillonite). Clays are said to be "inactive" $\left(A_{c}<0.75\right)$, "normal" $\left(A_{c}, 0.75-\right.$ $1.25)$, and "active" $\left(A_{c}>1.25\right)$.

\section{ANALYTICAL PROCEDURE}

Testing of the samples for their Atterberg limits was performed on board the Glomar Challenger immediately after the cores were sampled. A sample was placed in a preweighed aluminum moisture dish, weighed on a triple-beam balance, and placed in a drying oven at $110^{\circ} \mathrm{C}$ for drying overnight. Upon removal from the oven, the sample was immediately reweighed and water loss determined. At the same time, liquid limits were determined. Half of the sample was taken to determine its water content; the other half was used for plastic limit analysis.

Liquid limits were determined according to the simplified procedure described by Lambe (1951) and calculated as follows:

$$
W_{L}=w_{N}\left(\frac{N}{25}\right)^{0.121}
$$

where $w_{N}=$ the water content of the sediment sample and $N=$ the number of drops of a standard liquid limit cup required to close the groove in the sediment sample. Values of liquid limit (Table 1) represent an average of three separate determinations of the same sample.

Samples were also taken for analysis of organic carbon content and particle size distribution. Some of the carbon analyses were performed on ship, others were done later at the University of Oklahoma laboratory. All were analyzed in a Leco combustion apparatus. Grain size analysis was done at the DSDP laboratory in La Jolla.

\section{Quaternary Sediments}

The mantle of Quaternary, hemipelagic mud of the Guatemalan continental margin thins seaward and contains significant quantities of siliceous and calcareous fossil remains. Sediment thickness varies from greater than 200 meters at Site $\mathbf{4 9 6}$ on the upper trench slope to approximately 100 meters at Site 498 on the lower trench slope. Within the Middle America Trench the thickness of Quaternary sediments is variable, ranging from about 80 meters (Site 500) to 120 meters (Site 499). These sediments are interbedded pelagic diatomaceous muds and fine sand, and muddy turbidites. Quaternary sediments are only about 60 meters thick at Site 495 on the oceanic plate and are composed of diatomaceous hemipelagic muds. Sedimentation rates vary from 300 $\mathrm{m} / \mathrm{m}$.y, in the trench (Site 499 ) to $37 \mathrm{~m} / \mathrm{m}$.y. on the oceanic plate (Site 495). As expected, the greatest sediment accumulation occurs in the trench, because it is the ultimate resting place for sediment transferred from higher elevations on the slope. Dominant clay minerals are illite, montmorillonite, and kaolinite. The latter two are most abundant (Heinemann, this volume). Illite is missing from Site 495 on the ocean plate.

Organic carbon contents of the samples ranged from $0.62 \%$ to $3.59 \%$, averaging $2.00 \%(\mathrm{~s}=0.62 \%, \mathrm{n}=52)$.

The data in Table 1 indicate that the majority of the sediments analyzed are classified as "organic clays of medium to high plasticity, containing micaceous sands and silts" ( $\mathrm{OH}$ soils, as defined by the Unified Soil Clas- 
Table 1. Summary of sediment properties (Atterberg limits, initial water content, particle size analysis, and activity of samples analyzed).

\begin{tabular}{|c|c|c|c|c|c|c|c|c|c|c|c|c|c|c|}
\hline \multirow{2}{*}{$\begin{array}{c}\text { Sample } \\
\text { (interval in } \mathrm{cm} \text { ) }\end{array}$} & \multirow{2}{*}{$\begin{array}{l}\text { Sub-bottom } \\
\text { Depth } \\
\text { (m) }\end{array}$} & \multicolumn{5}{|c|}{ Atterberg Limits } & \multicolumn{4}{|c|}{ Size Analysis } & \multicolumn{2}{|r|}{ Activity } & \multirow[b]{2}{*}{ Plasticity } & \multirow[b]{2}{*}{$\mathrm{OC} \%$} \\
\hline & & $w_{L}$ & $w_{P}$ & $I_{P}$ & $I_{L}$ & $w$ & Sand & Silt & Clay & Classification & $A_{c}$ & Classification & & \\
\hline \multicolumn{15}{|l|}{ Hole 494} \\
\hline $\begin{array}{l}1-1,133-135 \\
1-3,109-111 \\
1-5,69-71 \\
2-3,103-105 \\
2-6,119-121 \\
3-3,99-101 \\
3-6,118-120 \\
4-4,77-79\end{array}$ & $\begin{array}{r}1.30 \\
4.10 \\
6.70 \\
12.04 \\
16.70 \\
52.50 \\
27.20 \\
33.25\end{array}$ & $\begin{array}{r}127.1 \\
117.8 \\
113.0 \\
116.7 \\
76.3 \\
121.4 \\
117.7 \\
138.7\end{array}$ & $\begin{array}{l}45.9 \\
56.6 \\
50.0 \\
48.5 \\
56.4 \\
54.7 \\
57.3 \\
63.3\end{array}$ & $\begin{array}{l}81.2 \\
61.2 \\
63.0 \\
68.2 \\
19.9 \\
66.7 \\
60.4 \\
75.4\end{array}$ & $\begin{array}{l}0.77 \\
0.49 \\
1.10 \\
0.82 \\
0.59 \\
0.55 \\
0.52 \\
0.37\end{array}$ & $\begin{array}{r}108.4 \\
86.6 \\
100.0 \\
104.6 \\
68.2 \\
91.2 \\
88.6 \\
91.1\end{array}$ & $\begin{array}{r}3.4 \\
0.8 \\
15.5 \\
11.3 \\
12.7 \\
8.7 \\
81.9 \\
11.9 \\
0.7\end{array}$ & $\begin{array}{l}47.6 \\
36.9 \\
41.8 \\
41.5 \\
45.9 \\
46.1 \\
46.3 \\
40.1\end{array}$ & $\begin{array}{l}49.0 \\
62.4 \\
42.7 \\
47.1 \\
41.4 \\
45.2 \\
41.8 \\
59.2\end{array}$ & $\begin{array}{l}\text { Silty clay } \\
\text { Silty clay } \\
\text { Silty clay } \\
\text { Silty clay } \\
\text { Clayey silt } \\
\text { Clayey silt } \\
\text { Clayey silt } \\
\text { Silty clay }\end{array}$ & $\begin{array}{l}1.66 \\
0.98 \\
1.47 \\
1.45 \\
0.48 \\
1.47 \\
1.44 \\
1.27\end{array}$ & $\begin{array}{l}\text { Active } \\
\text { Normal } \\
\text { Active } \\
\text { Active } \\
\text { Inactive } \\
\text { Active } \\
\text { Active } \\
\text { Active }\end{array}$ & $\begin{array}{l}\mathrm{CH} \\
\mathrm{OH} \\
\mathrm{OH} \\
\mathrm{OH} \\
\mathrm{OH} \\
\mathrm{OH} \\
\mathrm{OH} \\
\mathrm{OH}\end{array}$ & $\begin{array}{l}2.54 \\
1.65 \\
2.25 \\
2.12 \\
1.43 \\
2.32 \\
2.42 \\
2.77\end{array}$ \\
\hline $\begin{array}{l}\text { Mean }(\bar{x}) \\
\text { Std. Dev. (s) }\end{array}$ & & $\begin{array}{r}116.1 \\
17.9\end{array}$ & $\begin{array}{r}54.1 \\
5.6\end{array}$ & $\begin{array}{l}62.0 \\
18.5\end{array}$ & $\begin{array}{l}0.65 \\
0.23\end{array}$ & $\begin{array}{l}92.3 \\
12.5\end{array}$ & $\begin{array}{l}8.1 \\
5.7\end{array}$ & $\begin{array}{r}43.3 \\
3.8\end{array}$ & $\begin{array}{r}48.6 \\
8.0\end{array}$ & & $\begin{array}{l}1.28 \\
0.38\end{array}$ & & & $\begin{array}{l}2.19 \\
0.45\end{array}$ \\
\hline \multicolumn{15}{|l|}{ Hole 494A } \\
\hline $\begin{array}{l}1-1,102-104 \\
1-3,70-72 \\
2-7,37-39 \\
5-1,40-42\end{array}$ & $\begin{array}{r}1.00 \\
3.70 \\
17.90 \\
38.40\end{array}$ & $\begin{array}{r}111.6 \\
113.4 \\
108.8 \\
98.1\end{array}$ & $\begin{array}{l}56.6 \\
56.6 \\
59.1 \\
53.7\end{array}$ & $\begin{array}{l}55.0 \\
56.8 \\
49.8 \\
44.4\end{array}$ & $\begin{array}{l}0.52 \\
0.40 \\
0.46 \\
0.16\end{array}$ & $\begin{array}{l}85.0 \\
79.3 \\
64.2 \\
46.5\end{array}$ & $\begin{array}{l}0.4 \\
1.8\end{array}$ & $\begin{array}{l}38.6 \\
46.0\end{array}$ & $\begin{array}{l}61.0 \\
52.2\end{array}$ & $\begin{array}{l}\text { Silty clay } \\
\text { Silty clay }\end{array}$ & $\begin{array}{l}1.33 \\
\\
0.82 \\
0.85\end{array}$ & $\begin{array}{l}\text { Active } \\
\text { Normal } \\
\text { Normal }\end{array}$ & $\begin{array}{l}\mathrm{OH} \\
\mathrm{OH} \\
\mathrm{OH} \\
\mathrm{OH}\end{array}$ & $\begin{array}{l}2.27 \\
2.12 \\
2.15 \\
1.93\end{array}$ \\
\hline $\begin{array}{l}\text { Mean }(\bar{x}) \\
\text { Std. Dev. (s) }\end{array}$ & & $\begin{array}{r}107.9 \\
6.8\end{array}$ & $\begin{array}{r}56.5 \\
2.2\end{array}$ & $\begin{array}{r}51.5 \\
5.6\end{array}$ & $\begin{array}{l}0.39 \\
0.16\end{array}$ & $\begin{array}{l}68.8 \\
17.2\end{array}$ & $\begin{array}{l}2.4 \\
2.4\end{array}$ & $\begin{array}{r}46.1 \\
7.5\end{array}$ & $\begin{array}{r}51.5 \\
9.9\end{array}$ & & $\begin{array}{l}1.00 \\
0.29\end{array}$ & & & $\begin{array}{l}2.12 \\
0.14\end{array}$ \\
\hline \multicolumn{15}{|l|}{ Hole 495} \\
\hline $\begin{array}{l}3-1,67-70 \\
3-3,115-117 \\
3-6,132-134 \\
4-3,140-143 \\
4-5,63-66 \\
5-1,17-21 \\
5-3,128-132 \\
5-5,127-131 \\
6-4,59-63\end{array}$ & $\begin{array}{l}19.70 \\
23.16 \\
27.83 \\
32.92 \\
35.13 \\
38.17 \\
42.30 \\
45.30 \\
52.61\end{array}$ & $\begin{array}{l}113.5 \\
165.8 \\
117.5 \\
171.5 \\
178.9 \\
151.3 \\
135.2 \\
166.7 \\
147.2\end{array}$ & $\begin{array}{l}52.7 \\
88.0 \\
84.0 \\
81.0 \\
78.3 \\
80.7 \\
65.3 \\
85.1 \\
78.1\end{array}$ & \begin{tabular}{r|r}
60.8 & \\
77.8 \\
93.5 \\
101.9 \\
109.6 \\
70.6 \\
69.9 \\
81.6 \\
69.1
\end{tabular} & $\begin{array}{l}0.60 \\
1.12 \\
0.74 \\
0.67 \\
0.63 \\
1.02 \\
1.19 \\
0.78 \\
0.89\end{array}$ & $\begin{array}{r}89.5 \\
175.4 \\
153.3 \\
149.0 \\
148.9 \\
152.5 \\
88.7 \\
148.7 \\
139.5\end{array}$ & $\begin{array}{l}3.5 \\
1.1 \\
0.4 \\
0.7 \\
0.6 \\
1.8 \\
2.4 \\
2.0 \\
3.8\end{array}$ & $\begin{array}{l}57.9 \\
24.8 \\
37.0 \\
27.3 \\
31.3 \\
39.8 \\
37.2 \\
40.3 \\
53.1\end{array}$ & $\begin{array}{l}38.6 \\
74.1 \\
62.6 \\
71.9 \\
68.1 \\
58.4 \\
60.4 \\
57.7 \\
43.1\end{array}$ & $\begin{array}{l}\text { Clayey silt } \\
\text { Silty clay } \\
\text { Silty clay } \\
\text { Silty clay } \\
\text { Silty clay } \\
\text { Silty clay } \\
\text { Silty clay } \\
\text { Silty clay } \\
\text { Clayey silt }\end{array}$ & $\begin{array}{l}1.57 \\
1.05 \\
1.49 \\
1.42 \\
1.61 \\
1.21 \\
1.16 \\
1.41 \\
1.60\end{array}$ & $\begin{array}{l}\text { Active } \\
\text { Normal } \\
\text { Active } \\
\text { Active } \\
\text { Active } \\
\text { Normal } \\
\text { Normal } \\
\text { Active } \\
\text { Active }\end{array}$ & $\begin{array}{l}\mathrm{OH} \\
\mathrm{OH} \\
\mathrm{OH} \\
\mathrm{OH} \\
\mathrm{OH} \\
\mathrm{OH} \\
\mathrm{OH} \\
\mathrm{OH} \\
\mathrm{OH} \\
\mathrm{OH}\end{array}$ & $\begin{array}{l}0.62 \\
0.76 \\
2.13 \\
-\overline{15} \\
1.15 \\
0.86 \\
0.89 \\
1.45 \\
1.22\end{array}$ \\
\hline $\begin{array}{l}\text { Mean }(\bar{x}) \\
\text { Std. Dev. (s) }\end{array}$ & & $\begin{array}{r}156.4 \\
21.7\end{array}$ & $\begin{array}{l}77.0 \\
11.2\end{array}$ & $\begin{array}{l}81.6 \\
16.6\end{array}$ & $\begin{array}{l}0.85 \\
0.22\end{array}$ & $\begin{array}{r}138.4 \\
29.5\end{array}$ & $\begin{array}{l}1.8 \\
1.2\end{array}$ & $\begin{array}{l}38.7 \\
10.9\end{array}$ & $\begin{array}{l}59.4 \\
12.0\end{array}$ & & $\begin{array}{l}1.39 \\
0.21\end{array}$ & & & $\begin{array}{l}1.14 \\
0.48\end{array}$ \\
\hline \multicolumn{15}{|l|}{ Hole 496} \\
\hline $\begin{array}{l}1-1,96-99 \\
2-1,130-132 \\
5-6,129-131 \\
6-5,110-112 \\
8-1,70-74 \\
9-5,80-84 \\
10-5,80-84 \\
12-5,20-25 \\
13-3,60-62 \\
14-2,100-102 \\
16-1,120-122 \\
17-1,80-82 \\
19-3,75-78 \\
20-2,102-104 \\
21-6,138-140 \\
22-6,100-102\end{array}$ & $\begin{array}{r}0.98 \\
10.81 \\
46.80 \\
52.61 \\
65.22 \\
80.82 \\
90.32 \\
108.72 \\
115.61 \\
124.01 \\
141.71 \\
150.81 \\
172.76 \\
181.03 \\
196.89 \\
206.01\end{array}$ & $\begin{array}{l}127.8 \\
126.2 \\
142.6 \\
118.7 \\
145.0 \\
112.6 \\
108.4 \\
139.9 \\
130.5 \\
106.5 \\
136.2 \\
117.7 \\
124.2 \\
129.2 \\
118.4 \\
123.2\end{array}$ & $\begin{array}{l}52.9 \\
62.3 \\
72.3 \\
57.5 \\
49.8 \\
46.4 \\
55.4 \\
64.4 \\
60.1 \\
56.9 \\
74.2 \\
57.9 \\
67.3 \\
49.7 \\
58.1 \\
58.9\end{array}$ & $\begin{array}{l}74.9 \\
63.9 \\
70.3 \\
61.1 \\
95.2 \\
66.2 \\
53.1 \\
75.4 \\
70.4 \\
49.8 \\
61.9 \\
59.8 \\
56.9 \\
79.5 \\
60.3 \\
64.4 \\
\end{array}$ & $\begin{array}{l}1.18 \\
0.61 \\
0.54 \\
0.55 \\
0.55 \\
0.87 \\
0.64 \\
0.83 \\
0.49 \\
0.60 \\
0.25 \\
0.55 \\
0.33 \\
0.50 \\
0.36 \\
0.44\end{array}$ & $\begin{array}{r}141.2 \\
101.0 \\
110.8 \\
90.9 \\
102.0 \\
104.4 \\
89.4 \\
89.5 \\
94.6 \\
86.2 \\
89.4 \\
90.9 \\
86.2 \\
89.7 \\
79.9 \\
87.0\end{array}$ & $\begin{array}{l}0.6 \\
1.6 \\
2.2 \\
4.1 \\
2.1\end{array}$ & $\begin{array}{l}92.8 \\
59.2 \\
48.4 \\
55.9 \\
49.7\end{array}$ & $\begin{array}{r}6.5 \\
39.2 \\
49.4 \\
40.0 \\
48.3\end{array}$ & $\begin{array}{l}\text { Silt } \\
\text { Clayey silt } \\
\text { Silty clay } \\
\text { Clayey silt } \\
\text { Clayey silt }\end{array}$ & $\begin{array}{l}9.52 \\
1.52 \\
1.15 \\
1.98 \\
1.25\end{array}$ & $\begin{array}{l}\text { Active } \\
\text { Active } \\
\text { Normal } \\
\text { Active } \\
\text { Active }\end{array}$ & $\begin{array}{l}\mathrm{OH} \\
\mathrm{OH} \\
\mathrm{OH} \\
\mathrm{OH} \\
\mathrm{CH} \\
\mathrm{OH} \\
\mathrm{OH} \\
\mathrm{OH} \\
\mathrm{OH} \\
\mathrm{OH} \\
\mathrm{OH} \\
\mathrm{OH} \\
\mathrm{OH} \\
\mathrm{OH} \\
\mathrm{OH} \\
\mathrm{OH} \\
\mathrm{OH}\end{array}$ & $\begin{array}{l}2.82 \\
\overline{-} \\
1.94 \\
2.50 \\
2.47 \\
1.83 \\
2.50 \\
1.95 \\
3.59 \\
2.35 \\
2.38 \\
3.09 \\
2.20 \\
2.06\end{array}$ \\
\hline $\begin{array}{l}\text { Mean }(\bar{x}) \\
\text { Std. Dev. (s) }\end{array}$ & & $\begin{array}{r}125.4 \\
11.6\end{array}$ & $\begin{array}{r}59.0 \\
7.8\end{array}$ & $\begin{array}{l}66.4 \\
11.2\end{array}$ & $\begin{array}{l}0.58 \\
0.23\end{array}$ & $\begin{array}{l}95.8 \\
14.5\end{array}$ & $\begin{array}{l}1.7 \\
1.2\end{array}$ & $\begin{array}{l}56.7 \\
15.5\end{array}$ & $\begin{array}{l}43.8 \\
16.3\end{array}$ & & $\begin{array}{l}2.50 \\
2.85\end{array}$ & & & $\begin{array}{l}2.44 \\
0.50\end{array}$ \\
\hline \multicolumn{15}{|l|}{ Hole 497} \\
\hline $\begin{array}{l}1-1,98-102 \\
2-1,138-140 \\
3-3,60-64 \\
4-1,110-112 \\
5-3,80-84 \\
6-1,40-42 \\
7-4,100-102 \\
9-3,80-82 \\
10-4,30-32 \\
12-6,76-78 \\
13-3,93-100 \\
14-3,63-65 \\
16-8,30-32\end{array}$ & $\begin{array}{r}1.01 \\
8.39 \\
20.12 \\
27.11 \\
40.32 \\
45.41 \\
65.01 \\
77.31 \\
87.81 \\
110.27 \\
115.50 \\
124.64 \\
150.81\end{array}$ & $\begin{array}{l}113.5 \\
132.4 \\
126.4 \\
112.9 \\
104.4 \\
124.9 \\
100.4 \\
111.2 \\
145.7 \\
123.7 \\
112.2 \\
137.9 \\
149.9\end{array}$ & $\begin{array}{l}40.4 \\
48.6 \\
38.7 \\
43.2 \\
39.5 \\
52.5 \\
54.9 \\
56.3 \\
70.0 \\
72.0 \\
57.1 \\
69.9 \\
66.6\end{array}$ & $\begin{array}{l}73.1 \\
83.8 \\
87.6 \\
69.7 \\
64.9 \\
72.4 \\
45.4 \\
54.9 \\
75.7 \\
51.7 \\
55.0 \\
67.8 \\
83.4\end{array}$ & $\begin{array}{l}1.39 \\
1.05 \\
0.84 \\
1.00 \\
0.94 \\
0.76 \\
0.66 \\
0.74 \\
0.35 \\
0.61 \\
0.58 \\
0.38 \\
0.36\end{array}$ & $\begin{array}{r}142.4 \\
136.5 \\
112.3 \\
112.9 \\
100.3 \\
107.3 \\
85.1 \\
96.8 \\
96.5 \\
103.5 \\
112.7 \\
95.7 \\
96.3\end{array}$ & $\begin{array}{l}4.5 \\
2.6 \\
2.5 \\
1.7 \\
1.7 \\
2.9\end{array}$ & $\begin{array}{l}46.7 \\
50.9 \\
64.5 \\
56.4 \\
50.7 \\
56.3\end{array}$ & $\begin{array}{l}48.8 \\
46.5 \\
33.0 \\
42.0 \\
47.7 \\
40.7\end{array}$ & $\begin{array}{l}\text { Silty clay } \\
\text { Clayey silt } \\
\text { Clayey silt } \\
\text { Clayey silt } \\
\text { Clayey silt } \\
\text { Clayey silt }\end{array}$ & $\begin{array}{l}1.49 \\
1.80 \\
2.65 \\
1.66 \\
1.36 \\
1.78\end{array}$ & $\begin{array}{l}\text { Active } \\
\text { Active } \\
\text { Active } \\
\text { Active } \\
\text { Active } \\
\text { Active }\end{array}$ & $\begin{array}{l}\mathrm{CH} \\
\mathrm{CH} \\
\mathrm{CH} \\
\mathrm{CH} \\
\mathrm{CH} \\
\mathrm{OH} \\
\mathrm{OH} \\
\mathrm{OH} \\
\mathrm{OH} \\
\mathrm{OH} \\
\mathrm{OH} \\
\mathrm{OH} \\
\mathrm{OH}\end{array}$ & $\begin{array}{l}2.57 \\
2.92 \\
2.19 \\
1.85 \\
2.20 \\
2.63 \\
1 . \overline{1} \\
2.11 \\
2.32 \\
2.44 \\
1.01\end{array}$ \\
\hline $\begin{array}{l}\text { Mean }(\bar{x}) \\
\text { Std. Dev. (s) }\end{array}$ & & $\begin{array}{r}122.7 \\
15.5\end{array}$ & $\begin{array}{l}54.6 \\
12.2\end{array}$ & $\begin{array}{l}68.1 \\
13.2\end{array}$ & $\begin{array}{l}0.74 \\
0.30\end{array}$ & $\begin{array}{r}107.6 \\
16.4\end{array}$ & $\begin{array}{l}2.3 \\
0.9\end{array}$ & $\begin{array}{r}52.8 \\
5.2\end{array}$ & $\begin{array}{r}44.9 \\
5.3\end{array}$ & & $\begin{array}{l}1.62 \\
0.42\end{array}$ & & & $\begin{array}{l}2.17 \\
0.52\end{array}$ \\
\hline \multicolumn{15}{|l|}{ Hole 499} \\
\hline $\begin{array}{l}1-1,138-140 \\
2-4,78-80 \\
3-4,98-102 \\
5-5,138-140 \\
6-4,28-30 \\
7-2,70-72 \\
9, C C, 10-12\end{array}$ & $\begin{array}{r}1.39 \\
6.79 \\
16.01 \\
36.89 \\
43.79 \\
50.71 \\
76.11\end{array}$ & $\begin{array}{r}63.5 \\
130.1 \\
117.3 \\
105.8 \\
80.9 \\
110.2 \\
74.3\end{array}$ & $\begin{array}{l}38.4 \\
58.7 \\
51.3 \\
51.0 \\
39.3 \\
51.2 \\
36.7\end{array}$ & $\begin{array}{l}25.1 \\
71.4 \\
66.0 \\
54.8 \\
41.6 \\
58.9 \\
37.6\end{array}$ & $\begin{array}{l}1.25 \\
0.97 \\
0.80 \\
0.73 \\
1.29 \\
0.64 \\
1.22\end{array}$ & \begin{tabular}{r|}
69.9 \\
128.0 \\
104.2 \\
91.0 \\
93.1 \\
89.0 \\
82.6
\end{tabular} & $\begin{array}{r}0.5 \\
3.2 \\
0.6 \\
5.6 \\
16.8 \\
4.0 \\
22.3\end{array}$ & $\begin{array}{l}70.1 \\
55.7 \\
76.4 \\
56.6 \\
43.4 \\
63.4 \\
42.9\end{array}$ & $\begin{array}{l}29.4 \\
41.2 \\
23.0 \\
37.8 \\
39.8 \\
32.6 \\
34.8\end{array}$ & $\begin{array}{l}\text { Clayey silt } \\
\text { Clayey silt } \\
\text { Silt } \\
\text { Clayey silt } \\
\text { Clayey silt } \\
\text { Clayey silt } \\
\text { Sand-silt- } \\
\text { clay }\end{array}$ & $\begin{array}{l}0.85 \\
1.73 \\
2.87 \\
1.45 \\
1.04 \\
1.81 \\
1.08\end{array}$ & $\begin{array}{l}\text { Normal } \\
\text { Active } \\
\text { Active } \\
\text { Active } \\
\text { Normal } \\
\text { Active } \\
\text { Normal }\end{array}$ & $\begin{array}{l}\mathrm{OH} \\
\mathrm{OH} \\
\mathrm{OH} \\
\mathrm{OH} \\
\mathrm{OH} \\
\mathrm{OH} \\
\mathrm{OH}\end{array}$ & $\begin{array}{l}1.07 \\
2.39 \\
1.94 \\
1.88 \\
1.39 \\
1.27 \\
1.95\end{array}$ \\
\hline $\begin{array}{l}12-3,130-132 \\
14-3,18-20 \\
15-2,138-142 \\
17-3,60-62 \\
19-4,82-84\end{array}$ & $\begin{array}{l}100.31 \\
118.19 \\
127.40 \\
138.11 \\
167.83\end{array}$ & $\begin{array}{l}143.9 \\
120.1 \\
125.9 \\
125.6 \\
142.2\end{array}$ & $\begin{array}{l}70.7 \\
58.9 \\
70.7 \\
71.6 \\
49.0\end{array}$ & $\begin{array}{l}73.2 \\
61.2 \\
55.2 \\
54.1 \\
93.2\end{array}$ & $\begin{array}{l}0.32 \\
0.40 \\
0.56 \\
0.72 \\
0.58\end{array}$ & $\begin{array}{r}93.9 \\
83.6 \\
102.6 \\
110.7 \\
102.8\end{array}$ & $\begin{array}{l}1.6 \\
0.9 \\
2.7 \\
1.5\end{array}$ & $\begin{array}{l}53.1 \\
38.4 \\
44.5 \\
34.6\end{array}$ & $\begin{array}{l}45.4 \\
60.7 \\
52.8 \\
63.8\end{array}$ & $\begin{array}{l}\text { Clayey silt } \\
\text { Silty clay } \\
\text { Silty clay } \\
\text { Silty clay }\end{array}$ & $\begin{array}{l}1.35 \\
0.91 \\
1.02 \\
1.46\end{array}$ & $\begin{array}{l}\text { Active } \\
\text { Normal } \\
\text { Normal } \\
\text { Active }\end{array}$ & $\begin{array}{l}\mathrm{OH} \\
\mathrm{OH} \\
\mathrm{OH} \\
\mathrm{OH} \\
\mathrm{CH}\end{array}$ & $\begin{array}{l}2.32 \\
1.95 \\
1.32 \\
1.43 \\
1.16\end{array}$ \\
\hline $\begin{array}{l}\text { Mean }(\bar{x}) \\
\text { Std. Dev. (s) }\end{array}$ & & $\begin{array}{r}111.6 \\
26.1\end{array}$ & $\begin{array}{l}53.9 \\
12.5\end{array}$ & $\begin{array}{l}57.7 \\
17.9\end{array}$ & $\begin{array}{l}0.79 \\
0.33\end{array}$ & $\begin{array}{l}95.9 \\
15.0\end{array}$ & $\begin{array}{l}5.4 \\
7.3\end{array}$ & $\begin{array}{l}52.6 \\
13.4\end{array}$ & $\begin{array}{l}41.9 \\
12.8\end{array}$ & & $\begin{array}{l}1.42 \\
0.58\end{array}$ & & & $\begin{array}{l}1.67 \\
045\end{array}$ \\
\hline
\end{tabular}

Note: Symbols: $w=$ initial water content ( $\%$ dry weight); $w_{L}=$ liquid limit; $w P=$ plastic limit; $I p=$ plasticity index; $I_{L}=$ liquidity index; $A_{C}=$ activity; $C H=$ inorganic clays of medium to high plasticity; $\mathrm{OH}=$ organic clays of medium to high plasticity containing micaceous sands and silts; and $\mathrm{OC} \%=$ percentage of organic carbon. 
sification, Wagner, 1957). Only eight samples, $14 \%$ of the total number analyzed, plotted above the A-line (Fig. 2), and those are classified as "inorganic clays of medium to high plasticity" ( $\mathrm{CH}$ soils, as defined by the Unified Soil Classification).

One might question the appropriateness of the Unified Soil Classification for classifying submarine sediments in view of the fact that it was developed specifically to classify terrestrial soils on the basis of their engineering characteristics. Application of this classification system may not be as inappropriate as may seem at first glance, inasmuch as many present-day terrestrial soils formed initially as marine deposits, and many others are secondarily derived from marine sediments. Moreover, classification on the basis of plasticity characteristics appears justifiable, because this property also relates to various mass transport mechanisms.

Figure 2 shows the position of each sample analyzed on the Casagrande Plasticity Chart. It is evident that those defined as "inorganic clays of medium to high plasticity" are primarily from Site 497, and Table 1 indicates that these sediments comprise a layer extending to a depth of 40.0 meters. No obvious textural or lithologic difference was observed in the cores taken below this depth.

Figure 3 shows the activity of the Guatemalan samples. All but one are considered to be either "normal" (15 samples) or "active" (34 samples).

\section{Implications of Plasticity for Sediment Transport}

Accumulation rates of the Quaternary sediments on the Guatemalan continental slope are quite high. At Site 496 , the rate is $205 \mathrm{~m} / \mathrm{m} . \mathrm{y}$.; at Site 497 , located in the Middle American Trench at the base of the inner trench slope wall, it is $300 \mathrm{~m} / \mathrm{m} . \mathrm{y}$. (von Huene, Aubouin, et al., 1980). Rapid downslope transport of sediment is clearly indicated, perhaps involving a variety of processes.

Because of the high plasticity index of the Quaternary sediments, mudflows, cohesive debris flows, or cohesive flows (defined on the basis of Bingham plastic flow behavior by Lowe, 1979) should be particularly effective in transferring sediment basinward.

Other mechanisms of subaqueous mass transport may likely contribute to the high sedimentation rates. An obvious mechanism is that of episodic turbidity currents, possibly generated from large-scale slumps initiated in the unconsolidated sediment column by tectonic events associated with a convergent continental margin.

\section{Low- Versus High-Latitude Plasticity Characteristics}

That the sediments off the Guatemalan continental margin are classified generally as $\mathrm{OH}$ soils (Wagner, 1957 ) is rather unusual when compared with the data available from other regions. Keller (1971), in a study of 67 surface sediment samples from the Norwegian and Greenland seas, indicates that they are largely inorganic clays of medium to high plasticity $(\mathrm{CH})$, sandy clays, and slightly plastic inorganic silts. Only two of the samples plotted below the A-line and were classified as organic clays (OH). Davie, Fenske, and Serocki (1978)

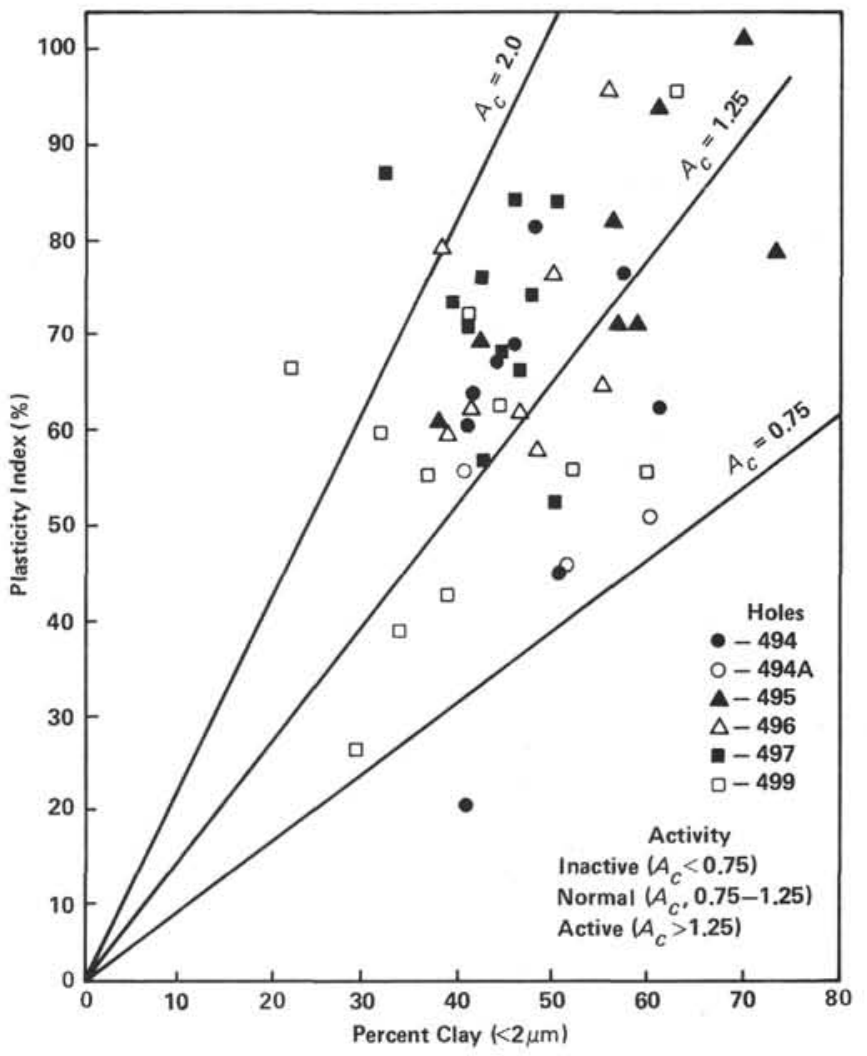

Figure 3. Activity chart showing activity of all samples analyzed.

show that clays generally lie above the A-line on a plasticity chart and are classed as $\mathrm{CH}$ soils. Siliceous oozes lie far below the A-line and are classified as $\mathrm{OH}$ soils, and calcareous oozes lie below the A-line and show low plasticity. Richards and Fager (1980) show that pelagic clays from 5600 meters in the Nares Abyssal Plain lie above the A-line and are classified as $\mathrm{CH}$ soils. On the other hand, Keller, Lambert, and Bennett (1979) find OH sediments covering a broad area of the lower continental slope of the east coast of the United States from the Wilmington Canyon south to Cape Hatteras.

Figure 4 shows the plasticity characteristics of sediment defined as terrigenous mud (T-2, JOIDES Panel on Sedimentary Petrology and Physical Properties, March 1974). These samples were taken on Leg 38 in the Norwegian-Greenland Sea and represent the author's as yet unpublished data. The sediments exhibit medium plasticity and lie, for the most part, above the A-line ( $\mathrm{CH}$ soils).

These sediments are composed of mixtures of montmorillonite, illite, kaolinite, and chlorite, with montmorillonite dominating (White, 1976). Consequently, they are not unlike the Guatemalan sediments with respect to clay mineral composition. However, organic carbon content is quite low, ranging between $0.0 \%$ to $1.3 \%$ (White, 1976). The Guatemalan sediments average $2.00 \%$ organic carbon; therefore their plasticity behavior appears to be significantly influenced by this component.

It is suggested that plasticity behavior of terrigenous and hemiterrigenous sediments in high-latitude regions 


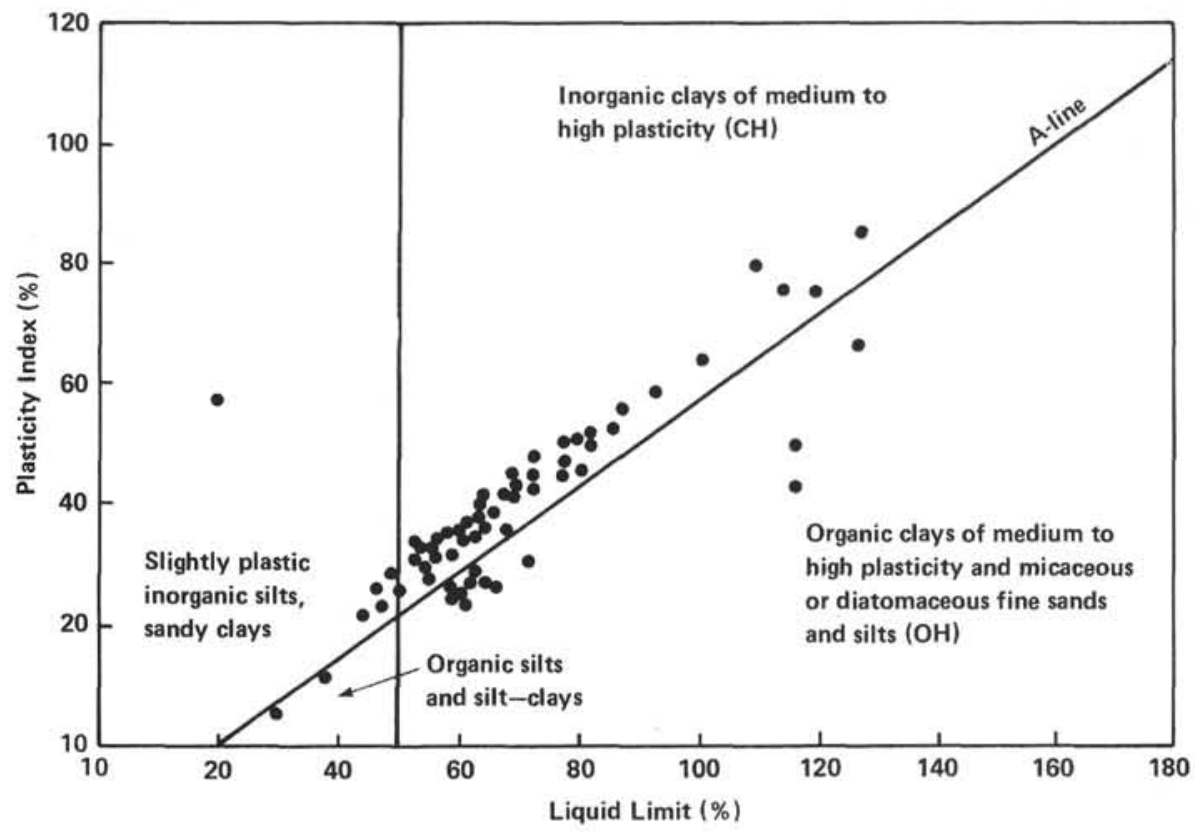

Figure 4. Casagrande Plasticity Chart showing plasticity characteristics of terrigenous (T-2) sediments from Leg 38, Norwegian-Greenland Sea (author's unpublished data).

is different from that of similar sediments accumulating in lower latitudes due to the greater amount of organic carbon (and soluble by-products) produced in these regions and incorporated in the sediments. Exceptions will occur (i.e., regions of upwelling), but organic matter content appears to be a factor in determining plasticity characteristics in compositionally similar sediments.

\section{CONCLUSIONS}

Although plasticity data for comparison are still rather limited, it is clear that the Guatemalan continental margin sediments are more organic and more compressible than similar types of sediments from higherlatitude areas. The only sediments showing comparable plasticity characteristics are those blanketing the lower continental slope of the eastern United States between the Wilmington Canyon and Cape Hatteras.

It appears likely that the high sedimentation rates determined for the Quaternary sediments of the Guatemalan continental margin are due, in part, to the highly plastic nature of the hemipelagic sediments. These sediments are located on a tectonically active continental margin and may be dominated by rheologically controlled downslope transport processes, including mudflows and cohesive debris flows.

\section{ACKNOWLEDGMENTS}

I thank William Harrison and Joseph Curiale for providing organic carbon analyses on the samples used for Atterberg limits. I also thank Bobb Carson, Richard Bennett, and Joseph Kravitz for their suggestions, which helped to improve this manuscript.

\section{REFERENCES}

Casagrande, A., 1948. Classification and identification of soils. Am. Soc, Eng. Trans, 113:901-931.

Davie, J. R., Fenske, C. W., and Serocki, S. T., 1978. Geotechnical properties of deep continental margin soils. Mar. Geotechnol., $3(1): 85-119$.

Keller, G. H., 1971. Engineering properties of Greenland and Norwegian basin sediments. In Proc. First Int. Conf. on Port and Ocean Engineering under Arctic Conditions, Vol. II, pp. 1285-1311.

Keller, G. H., Lamber, D. N., and Bennett, R. C., 1979. Geotechnical properties of continental slope deposits-Cape Hatteras to $\mathrm{Hy}$ drographer Canyon. Soc. Econ. Paleontol. Mineral. Spec. Publ. 27, pp. 131-151.

Lambe, T. W., 1951. Soil Testing for Engineers: New York (John Wiley \& Sons), p. 165.

Lowe, D. R., 1979. Sediment gravity flows: their classification and some problems of application to natural flows and deposits. Soc. Econ. Paleontol. Mineral. Spec. Publ. 27, pp. 75-82.

Richards, A. F., and Fager, E., 1980. Water content and Atterberg limits of sediments at DSDP Holes 417A and 418A, Legs 51 and 52, West Atlantic Ocean. In Robinson, P., Flower, M., Salisbury, M., et al., Init. Repts. DSDP, 51, 52, 53, Pt. 2: Washington (U.S. Govt. Printing Office), 1453-1455.

Skempton, A. W., 1953. Soil mechanics in relation to geology. Proc. Yorks. Geol. Soc., 29(Pt. 1, No. 3):33-62.

1970. The consolidation of clays by gravitational compaction. Q. J. Geol. Soc. London, 125:373-412.

von Huene, R., Aubouin, J., et al., 1980. Leg 67: the Deep Sea Drilling Project Mid-America Trench transect off Guatemala. Geol. Soc. Am. Bull., 91:421-432.

Wagner, A. A., 1957. The use of the Unified Soil Classification system by the Bureau of Reclamation. Proc. Int. Conf. Soil Mech. Found. Eng., 4th, 1:125.

White, S. M., 1976. Sediments of the Norwegian-Greenland Sea, DSDP Leg 38. In Talwani, M., Udintsev, G., et al., Init. Repts. DSDP, Suppl. to Vols. 38, 39, 40, and 41: Washington (U.S. Govt. Printing Office), 193-257. 